\title{
Generalized and inverse generalized synchronization of fractional-order discrete-time chaotic systems with non-identical dimensions
}

\author{
Amina-Aicha Khennaoui ${ }^{1}$, Adel Ouannas ${ }^{2}$, Samir Bendoukha ${ }^{3 *}$ (D) Giuseppe Grassi ${ }^{4}$, Xiong Wang ${ }^{5}$ and \\ Viet-Thanh Pham ${ }^{6}$
}

"Correspondence: sbendoukha@taibahu.edu.sa ${ }^{3}$ Department of Electrical Engineering, College of Engineering, Taibah University, Yanbu, Saudi Arabia Full list of author information is available at the end of the article

\section{Springer}

\begin{abstract}
In this paper, we introduce two approaches to the generalized synchronized synchronization and the inverse generalized synchronization of fractional discrete-time chaotic systems with non-identical dimensions. The convergence of the proposed approaches is established by means of recently developed stability theory. Numerical results are presented based on well-known maps in the literature. Two examples are considered: a 3D generalized synchronization and a 2D inverse generalized synchronization.
\end{abstract}

MSC: $34 \mathrm{A0}$; 34H10; 34D06

Keywords: Discrete chaos; Discrete fractional calculus; Generalized synchronization; Inverse generalized synchronization; Different dimensions

\section{Introduction}

The theory of fractional calculus is old as its inception can be attributed to two of the most prominent figures of modern calculus, L'Hôpital and Leibniz, as early as 1695 . The complete framework for this type of calculus was complete by the 1800s [1]. However, the same cannot be said about discrete fractional calculus, which has not seen the light of day until recently. Over the last decade, researchers have found interest in the theory as well as applications of discrete fractional calculus. In examining the theoretical literature, one observes a number of difficulties facing the subject. In fact, an exact definition of the fractional discrete operator itself has not yet been agreed upon. The general consensus seems to be restricted to the fact that unlike the integer operator, which is local, the fractional one has infinite memory. This is not at all unlike the Caputo operator in continuous time. A good description and analysis of this type of calculus can be found in [1]. Most of the notation adopted in our study is based on that of [1]. It is important to mention that discrete fractional calculus has been shown to provide more accurate models of natural phenomena. See, for instance, modeling the motion of a bead sliding on a wire [2]. The subject has also found application in optimal control [3].

(c) The Author(s) 2018. This article is distributed under the terms of the Creative Commons Attribution 4.0 International License (http://creativecommons.org/licenses/by/4.0/), which permits unrestricted use, distribution, and reproduction in any medium, provided you give appropriate credit to the original author(s) and the source, provide a link to the Creative Commons license, and indicate if changes were made. 
Among the interesting aspects of a discrete-time fractional system is its stability analysis. Obviously, the asymptotic stability of discrete fractional systems is an important subject since it brings us a step closer to establishing the existence of chaos and serves as a basis for proving the convergence of a system's states to zero (or some other equilibrium) as time approaches infinity. This is particularly important when dealing with the stabilization and synchronization of discrete fractional dynamical systems. Among the most interesting results reported in the literature are those of [4], where the author establishes bounds on the arguments of the eigenvalues related to the linearized system in order to guarantee asymptotic stability. Another interesting study is [5], where the authors established and proved a discrete fractional version of the well-known direct Lyapunov method. Some useful Lyapunov functions for Riemann-Liouville-like fractional difference equations can be looked up in [6]. More stability results can be found in [7, 8].

Chaos refers to the high sensitivity of a dynamical system to small changes in the initial condition. Discrete chaotic systems have been around for a while. Perhaps the most commonly studied and applied discrete chaotic system is the Hénon map, which was introduced in 1976 [9] as a discretization of the Poincaré section of the famous continuous-time Lorenz system. Soon after, numerous other maps were proposed and became of interest to researchers in the fields of communications and control including the Lozi system [10] and the 2-component flow model [11]. Generalizations of these 2-component systems to higher dimensions were proposed at a later stage including, for instance, the generalized Hénon map [12] and the Stefanski system [13]. This type of chaotic systems has found applications in many fields including engineering and science [14-17].

One of the most studied aspects of chaos is the synchronization of chaotic systems, which aim to force a slave system to mimic the dynamics of a master one. Numerous synchronization types and control strategies have been proposed throughout the years since Pecora et al. [18] had their first breakthrough. The synchronization of discrete-time systems has been studied by some researchers [19-27].

Fractional chaotic systems have recently become somewhat of a hot topic. Researchers have looked at the fractional counterparts for a number of conventional chaotic systems. Most recently, some advances have been made in relation to chaotic fractional discretetime systems and their applications [28-35]. The theory related to this kind of systems makes them suitable for certain applications such as encryption [36, 37]. Researchers in the field of secure communications and data encryption have suggested that fractional maps are more suitable than integer order ones as they include a new degree of freedom in their dependency on the fractional order. In addition, it has been reported that fractional maps have simpler forms yet hold richer dynamical behaviors. The synchronization of this type of systems remains to this day a new and mostly unexplored field. The references on the subject are only a few [37-43].

In this paper, we aim to propose control laws for two types of synchronization relating to fractional discrete-time systems, namely generalized synchronization (GS) and inverse generalized synchronization (IGS). GS is one of the most widely studied synchronization types. It refers to the existence of a functional relationship between the drive states and the response states [44]. Instead of the conventional definition of synchronization, which stipulates that the difference between the drive and response trajectories tends to zero as $t \rightarrow \infty$, GS forces the difference between the slave states and a function of the master states to zero. IGS is the natural reversal of GS, i.e., the error is the difference between 
the master states and a function of the slave states. The importance of GS and IGS stems from the fact that they can enrich the behavior of chaotic systems. This allows for more flexibility and has proven useful in many applications including secure communications [45]. Exact definitions of the GS and IGS types will be given in Sects. 3 and 4, respectively.

Due to the many reported advantages of fractional chaotic maps [30,33, 46], we found ourselves compelled to examine their synchronization. It is not always possible or desirable to completely synchronize two chaotic systems but rather to introduce a more generalized synchronization [44]. Our aim in this paper is to establish the convergence of the proposed GS and IGS schemes for a pair of fractional discrete systems by means of asymptotic stability results reported recently in the literature. The next section of this study will highlight some of the necessary notation and theory related to discrete fractional calculus and stability. Section 3 proposes the control law for the generalized synchronization of two fractional discrete-time systems with non-identical dimensions. Section 4 presents the control law for the inverse generalized synchronization following a similar approach. Section 5 presents the numerical results related to two particular examples. Finally, Sect. 6 provides a general summary of the main findings of this study.

\section{Basic concepts}

In this section, we aim to recall some of the theory required for the analysis throughout our paper. In particular, we will present some of the notation and definitions related to discrete fractional calculus and the stability of fractional discrete systems. Throughout our study, we will be using the notation and definitions adopted in [42] for discrete fractional calculus. We start by defining the $v$ th fractional sum of an arbitrary function $u: \mathbb{N}_{a} \rightarrow \mathbb{R}$ in the same way as postulated in [47], i.e.,

$$
\Delta_{a}^{-v} u(t)=\frac{1}{\Gamma(v)} \sum_{s=a}^{t-v}(t-\sigma(s))^{(v-1)} u(s)
$$

where $v>0$ and $\sigma(s)=s+1$. The notation $\mathbb{N}_{a}$ denotes the set of natural numbers starting from $a$ and $t^{v}$ denotes the falling function given by means of the gamma function $\Gamma$ as

$$
t^{(v)}=\frac{\Gamma(t+1)}{\Gamma(t+1-v)} .
$$

We also need to define the $v$ th order Caputo type delta difference of an arbitrary function $u$ defined over $\mathbb{N}_{a}$. According to [48], for $v \notin \mathbb{N}, t \in \mathbb{N}_{a+n-v}$, and $n=[v]+1$, this may be given by

$$
{ }^{C} \Delta_{a}^{v}=\Delta_{a}^{-(n-v)} \Delta^{n} u(t)=\frac{1}{\Gamma(n-v)} \sum_{s=a}^{t-(n-v)}(t-\sigma(s))^{(n-v-1)} \Delta^{n} u(s) .
$$

It is important to note that in the special case characterized by $0<v<1, n=1$ and $t \in$ $\mathbb{N}_{a+1-v}$ yielding

$$
{ }^{C} \Delta_{a}^{v} u(t)=\Delta_{a}^{-(1-v)} \Delta u(t)=\frac{1}{\Gamma(1-v)} \sum_{s=a}^{t-(1-v)}(t-\sigma(s))^{(-v)} \Delta_{s} u(s) .
$$


In order to be able to carry out the analysis and numerical simulations required in our paper, we need the following two theorems. The first theorem describes the equivalent discrete integral equation corresponding to a generic fractional discrete difference equation. This is needed to obtain the numerical formulas for the simulations. It is important to note that more recent work has been done on the subject of extending the well-known finite difference numerical method to fractional systems including, for instance, the work in [49]. The second theorem identifies the asymptotic stability condition for a generic fractional map.

Theorem 1 ([50]) The equivalent discrete integral equation to

$$
{ }^{C} \Delta_{a}^{v} u(t)=f(t+v-1, u(t+v-1))
$$

with $\Delta^{k} u=u_{k}, n=[v]+1$, and $k=0,1, \ldots, n-1$, can be formulated for $t \in \mathbb{N}_{a+n}$ as

$$
u(t)=u_{0}(t)+\frac{1}{\Gamma(v)} \sum_{s=a+n-v}^{t-v}(t-\sigma(s))^{(v-1)} f(s+v-1, u(s+v-1))
$$

where the initial iteration $u_{0}(t)$ reads

$$
u_{0}(t)=\sum_{k=0}^{m-1} \frac{(t-a)^{(k)}}{\Gamma(k+1)} \Delta^{k} u(a)
$$

Theorem $2([4])$ Given a vector-valued function $X(t)=\left(x_{1}(t), \ldots, x_{n}(t)\right)^{T}, 0<v \leq 1, A \in$ $R^{n \times n}$, and $\forall t \in \mathbb{N}_{a+1-v}$, the zero equilibrium of the linear fractional discrete-time system

$$
{ }^{C} \Delta_{a}^{v} X(t)=A X(t+v-1)
$$

is asymptotically stable if

$$
\lambda \in\left\{z \in \mathbb{C}:|z|<\left(2 \cos \frac{|\arg z|-\pi}{2-v}\right)^{v} \text { and }|\arg z|>\frac{v \pi}{2}\right\}
$$

for all the eigenvalues $\lambda$ of $A$.

\section{Generalized synchronization}

Let us consider the drive and response systems described by

$$
\left\{\begin{array}{l}
{ }^{C} \Delta_{a}^{v} X(t)=f(X(t+v-1)), \\
{ }^{C} \Delta_{a}^{v} Y(t)=B Y(t+v-1)+g(Y(t+v-1))+U,
\end{array} \quad \text { for } t \in \mathbb{N}_{a+1-v},\right.
$$

where $X(t)=\left(x_{1}(t), \ldots, x_{n}(t)\right)^{T}$ and $Y(t)=\left(y_{1}(t), \ldots, y_{m}(t)\right)^{T}$ are the corresponding state vectors, $0<v \leq 1, f: \mathbb{R}^{n} \rightarrow \mathbb{R}^{n}, B$ is an $m \times m$ matrix of parameters, $g: \mathbb{R}^{m} \rightarrow \mathbb{R}^{m}$ is a nonlinear function, and $U=\left(u_{i}\right)_{1 \leq i \leq m}$ is the vector controller to be determined by means of the synchronization process. In this section we are concerned with generalized synchronization, which is defined as follows. 
Definition 1 The pair (10) is said to be generalized synchronized with respect to the vector map $\phi$ if there exists a controller $U=\left(u_{i}\right)_{1 \leq i \leq m}$ and a map $\phi: \mathbb{R}^{n} \rightarrow \mathbb{R}^{m}$ such that

$$
\lim _{t \rightarrow+\infty}\|e(t):=Y(t)-\phi(X(t))\|=0
$$

Normally, in order to establish that a certain control law achieves a given type of synchronization, we must prove that the zero solution of the error system is asymptotically stable. Hence, the following theorem proposes a controller $U$ aimed at forcing the synchronization errors defined in (11) to zero asymptotically, i.e., as $t \rightarrow \infty$.

\section{Theorem 3 Subject to}

$$
\begin{aligned}
U(t)= & -C Y(t)-g(Y(t)) \\
& +(C-B) \phi(X(t))+{ }^{C} \Delta_{a}^{v} \phi(X(t)),
\end{aligned}
$$

where $t \in \mathbb{N}_{a+1-v}$,

$$
C=\left(\begin{array}{cccc}
c_{11} & b_{12} & \cdots & b_{1 m} \\
b_{21} & c_{22} & \cdots & b_{2 m} \\
\vdots & \vdots & \ddots & \vdots \\
b_{m 1} & b_{m 2} & \cdots & c_{m m}
\end{array}\right)
$$

and

$$
-2^{v}<b_{i i}-c_{i i}<0, \quad i=1,2, \ldots, m,
$$

the pair (10) is globally generalized synchronized with respect to $\phi$.

Proof The errors between the pair of systems (10) lead to

$$
{ }^{C} \Delta_{a}^{v} e(t)=B Y(t+v-1)+g(Y(t+v-1))-{ }^{C} \Delta_{a}^{v} \phi(X(t))+U .
$$

Substituting (12) into (15) yields the simplified form

$$
{ }^{C} \Delta_{a}^{v} e(t)=(B-C) e(t+v-1),
$$

where

$$
B-C=\left(\begin{array}{cccc}
b_{11}-c_{11} & 0 & \cdots & 0 \\
0 & b_{22}-c_{22} & \cdots & 0 \\
\vdots & \vdots & \ddots & \vdots \\
0 & 0 & \cdots & b_{m m}-c_{m m}
\end{array}\right)
$$

It is easy to see that the eigenvalues of $B-C$, which are simply $\lambda_{i}=b_{i i}-c_{i i}, i=1,2, \ldots, m$, satisfy the conditions

$$
\left|\lambda_{i}\right|<\left(2 \cos \frac{\left|\arg \lambda_{i}\right|-\pi}{2-v}\right)^{v}
$$


and

$$
\left|\arg \lambda_{i}\right|>\frac{v \pi}{2}
$$

It, therefore, follows immediately from Theorem 2 that the zero solution of (16) is asymptotically stable and the pair (10) is generalized synchronized.

\section{Inverse generalized synchronization}

Now, let us consider the drive and response chaotic systems of the form

$$
\left\{\begin{array}{l}
{ }^{C} \Delta_{a}^{v} X(t)=A X(t+v-1)+f(X(t+v-1)), \\
{ }^{C} \Delta_{a}^{v} Y(t)=g(Y(t+v-1))+U,
\end{array} \quad \text { for } t \in \mathbb{N}_{a+1-v},\right.
$$

where $X(t)=\left(x_{1}(t), \ldots, x_{n}(t)\right)^{T}$ and $Y(t)=\left(y_{1}(t), \ldots, y_{m}(t)\right)^{T}, 0<v \leq 1, A \in \mathbb{R}^{n \times n}, f: \mathbb{R}^{n} \rightarrow$ $\mathbb{R}^{n}$ is a nonlinear function, $g: \mathbb{R}^{m} \rightarrow \mathbb{R}^{m}$, and $U=\left(u_{i}\right)_{1 \leq i \leq m}$. Note here that the master and slave systems in (18) are of non-identical dimensions given by $n$ and $m$, respectively, such that $n \leq m$. In this section, we aim to introduce a control strategy that forces the slave states to synchronize with the master ones. The type of synchronization considered here is known as inverse generalized synchronization, which was considered in [51] for continuous-time fractional chaotic systems.

Definition 2 The pair (18) is said to be inverse generalized synchronized with respect to $\varphi$ if there exist a controller $U=\left(u_{i}\right)_{1 \leq i \leq m}$ and a map $\varphi: \mathbb{R}^{m} \longrightarrow \mathbb{R}^{n}$ such that

$$
\lim _{t \rightarrow+\infty}\|e(t):=X(t)-\varphi(Y(t))\|=0 .
$$

Suppose that $\varphi$ can be written as

$$
\varphi(Y(t))=H Y(t)+h(Y(t)),
$$

where $H \in \mathbb{R}^{n \times m}$ and $h: \mathbb{R}^{m} \rightarrow \mathbb{R}^{n}$ is a nonlinear function. The error dynamics between the drive and response systems (18) can be derived for $t \in \mathbb{N}_{a+1-v}$ as

$$
\begin{aligned}
{ }^{C} \Delta_{a}^{v} e(t)= & { }^{C} \Delta_{a}^{v} X(t)-{ }^{C} \Delta_{a}^{v} \varphi(Y(t)) \\
= & A X(t+v-1)+f(X(t+v-1))-H \times{ }^{C} \Delta_{a}^{v} Y(t)-{ }^{C} \Delta_{a}^{v} h(Y(t)) \\
= & A X(t+v-1)+f(X(t+v-1))-H \times g(Y(t+\beta-1)) \\
& -{ }^{C} \Delta_{a}^{v} h(Y(t))-H \times U .
\end{aligned}
$$

For $i>n$, we can simply choose

$$
u_{i}=0, \quad i=n+1, n+2, \ldots, m .
$$

This way, (21) can be written as follows:

$$
{ }^{C} \Delta_{a}^{v} e(t)=(A-L) e(t+v-1)+R-\hat{H} \times \hat{U}, \quad t \in \mathbb{N}_{a+1-v},
$$


where $\hat{U}=\left(u_{1}, \ldots, u_{n}\right)^{T}, \hat{H}$ is an invertible $n \times n$ matrix,

$$
R=L X(t)+(A-L) H Y(t)+(A-L) h(Y(t))+f(X(t))-H g(Y(t))-{ }^{C} \Delta_{a}^{v} h(Y(t)),
$$

and $L \in \mathbb{R}^{n \times n}$ is a control matrix chosen such that all the eigenvalues $\lambda_{i}, i=1, \ldots, n$, of $A-L$ satisfy the conditions

$$
\left|\lambda_{i}\right|<2^{v} \quad \text { and } \quad\left|\arg \left(\lambda_{i}\right)\right|=\pi, \quad i=1,2, \ldots, n .
$$

In order to achieve inverse generalized synchronization, our choice of the remaining control parameters can be made as follows:

$$
\hat{U}=\hat{H}^{-1} \times R
$$

where $\hat{H}^{-1} \in \mathbb{R}^{n \times n}$ is the inverse of matrix $\hat{H}$. By substituting (26) into (23), the error dynamics can be described as

$$
{ }^{C} \Delta_{a}^{v} e(t)=(A-L) e(t+v-1), \quad t \in \mathbb{N}_{a+1-v}
$$

With this dynamic, we can follow the same steps as in the proof of Theorem 3 to establish the following result.

Theorem 4 Subject to control laws (22) and (26) and by selecting the control matrix $L$ according to (25), the pair (18) is inverse generalized synchronized with respect to $\varphi$.

\section{Numerical results}

In order to put the control laws proposed in Theorems 3 and 4 to the test, we will present two numerical examples for a pair of fractional chaotic systems with different dimensions. In [33], the author introduced a complete fractional-order Hénon map based on the same discrete fractional calculus definitions adopted in our study. In addition, a fractional form of the 3D generalized Hénon map was proposed in [46] with the same notation used herein.

Before we go ahead and show our numerical results, let us first review some aspects of the drive and response systems. We consider as a drive system the 2D fractional Hénon map of the form

$$
\left\{\begin{array}{l}
{ }^{C} \Delta_{a}^{v} x_{1}(t)=x_{2}(t+v-1)+1-a_{1} x_{1}^{2}(t+v-1)-x_{1}(t+v-1), \\
{ }^{C} \Delta_{a}^{v} x_{2}(t)=b_{1} x_{1}(t+v-1)-x_{2}(t+v-1),
\end{array}\right.
$$

for $t \in \mathbb{N}_{a+1-v}$, which exhibits a chaotic attractor, for instance, when $\left(a_{1}, b_{1}\right)=(1.4,0.3)$, $a=0$, and $v=0.984$. The resulting chaotic attractor is shown in Fig. 1 and its general shape is similar to that of the integer order one. 


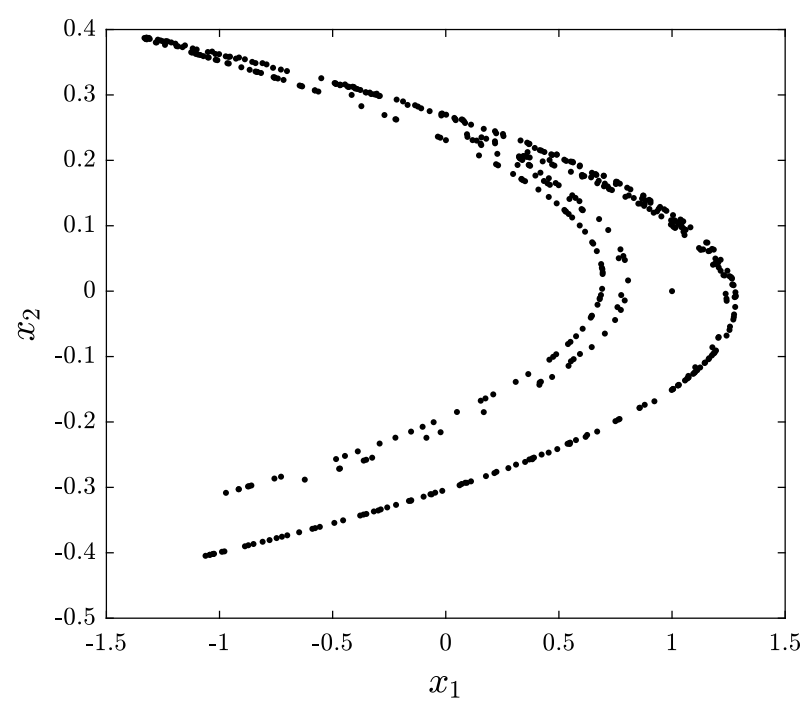

Figure 1 Phase space plot for the fractional Hénon map with $\left(a_{1}, b_{1}\right)=(1.4,0.3), v=0.984, a=0$, and $x_{1}(0)=x_{2}(0)=0$

As for the response system, we consider the 3D fractional generalized Hénon map. The system is described for $t \in \mathbb{N}_{a+1-v}$ as

$$
\left\{\begin{array}{l}
{ }^{C} \Delta_{a}^{v} y_{1}(t)=-y_{1}(t+v-1)-b_{2} y_{3}(t+v-1)+u_{1}(t+v-1), \\
{ }^{C} \Delta_{a}^{v} y_{2}(t)=b_{2} y_{3}(t+v-1)+y_{1}(t+v-1)-y_{2}(t+v-1)+u_{2}(t+v-1), \\
{ }^{C} \Delta_{a}^{v} y_{3}(t)=1+y_{2}(t+v-1)-a_{2} y_{3}^{2}(t+v-1)-y_{3}(t+v-1)+u_{3}(t+v-1),
\end{array}\right.
$$

where $u_{1}, u_{2}$, and $u_{3}$ are controllers. Subject to $\left(a_{2}, b_{2}\right)=(0.99,0.2), a=0$, and $v=0.984$, the uncontrolled map (29) with $u_{1}=u_{2}=u_{3}=0$ is chaotic as shown in Fig. 2. In the remainder of this section, we will put the findings of Theorems 3 and 4 to the test by considering two specific examples, a 3D generalized synchronization and a 2D inverse generalized synchronization.

\subsection{D generalized synchronization}

It is easy to see that the linear part of the response system (29) is given by

$$
B=\left(\begin{array}{ccc}
-1 & 0 & -b_{2} \\
1 & -1 & b_{2} \\
0 & 1 & -1
\end{array}\right) .
$$

Based on the proposed approach described in Sect. 3, the error system corresponding to the generalized synchronization strategy is defined as

$$
\left(e_{1}(t), e_{2}(t), e_{3}(t)\right)^{T}=\left(y_{1}(t), y_{2}(t), y_{3}(t)\right)^{T}-\phi\left(x_{1}(t), x_{2}(t)\right)
$$

where

$$
\phi\left(x_{1}(t), x_{2}(t)\right)=\left(x_{1}(t), x_{2}(t), x_{1}(t) x_{2}(t)\right)^{T} .
$$



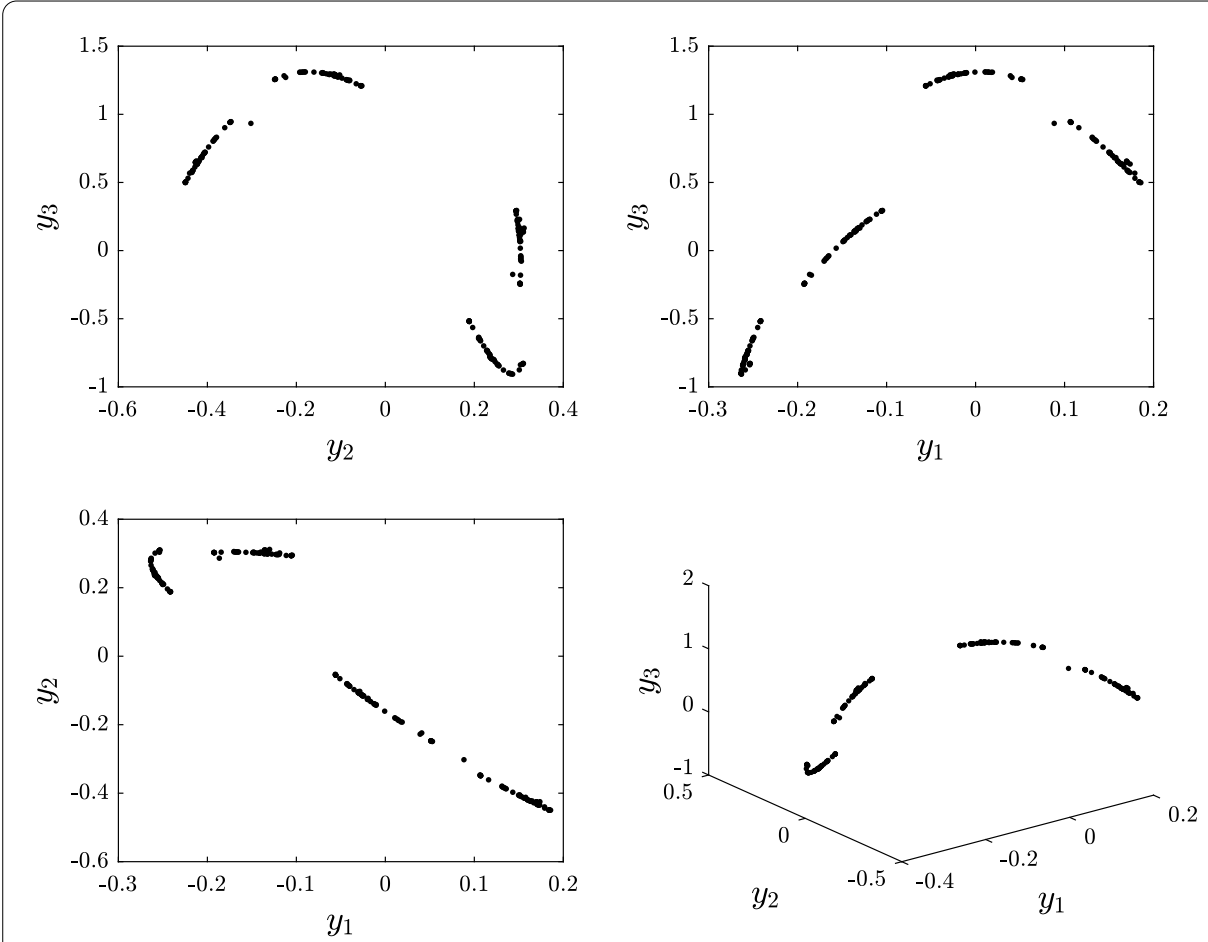

Figure 2 Phase portraits for the hyperchaotic Hénon map with $\left(a_{2}, b_{2}\right)=(0.99,0.2), v=0.984, a=0$, and $\left(y_{1}(0), y_{2}(0), y_{3}(0)\right)=(0.1,0.2,0.5)$

According to Theorem 3, there exists a control matrix $C$, which can be chosen as

$$
C=\left(\begin{array}{ccc}
0 & 0 & -b_{2} \\
1 & -\frac{1}{2} & b_{2} \\
0 & 1 & -\frac{2}{3}
\end{array}\right)
$$

which clearly satisfies conditions (13) and (14), and by extension systems (28) and (29) are generalized synchronized in 3D. Now that matrices $B$ and $C$ and function $\phi$ are known, it is rather easy to construct the control law according to (12). The resulting error system is of the form

$$
\left\{\begin{array}{l}
{ }^{C} \Delta_{a}^{v} e_{1}(t)=-e_{1}(t+v-1), \\
{ }^{C} \Delta_{a}^{v} e_{2}(t)=-\frac{1}{2} e_{2}(t+v-1), \\
{ }^{C} \Delta_{a}^{v} e_{3}(t)=-\frac{1}{3} e_{3}(t+v-1) .
\end{array}\right.
$$

The time evolution of the errors is depicted in Fig. 3. Clearly, synchronization is achieved as the errors converge to zero in sufficient time.

\subsection{D inverse generalized synchronization}

For the 2D inverse generalized scenario, the linear part of the slave system is

$$
A=\left(\begin{array}{cc}
-1 & 1 \\
b_{1} & -1
\end{array}\right)
$$



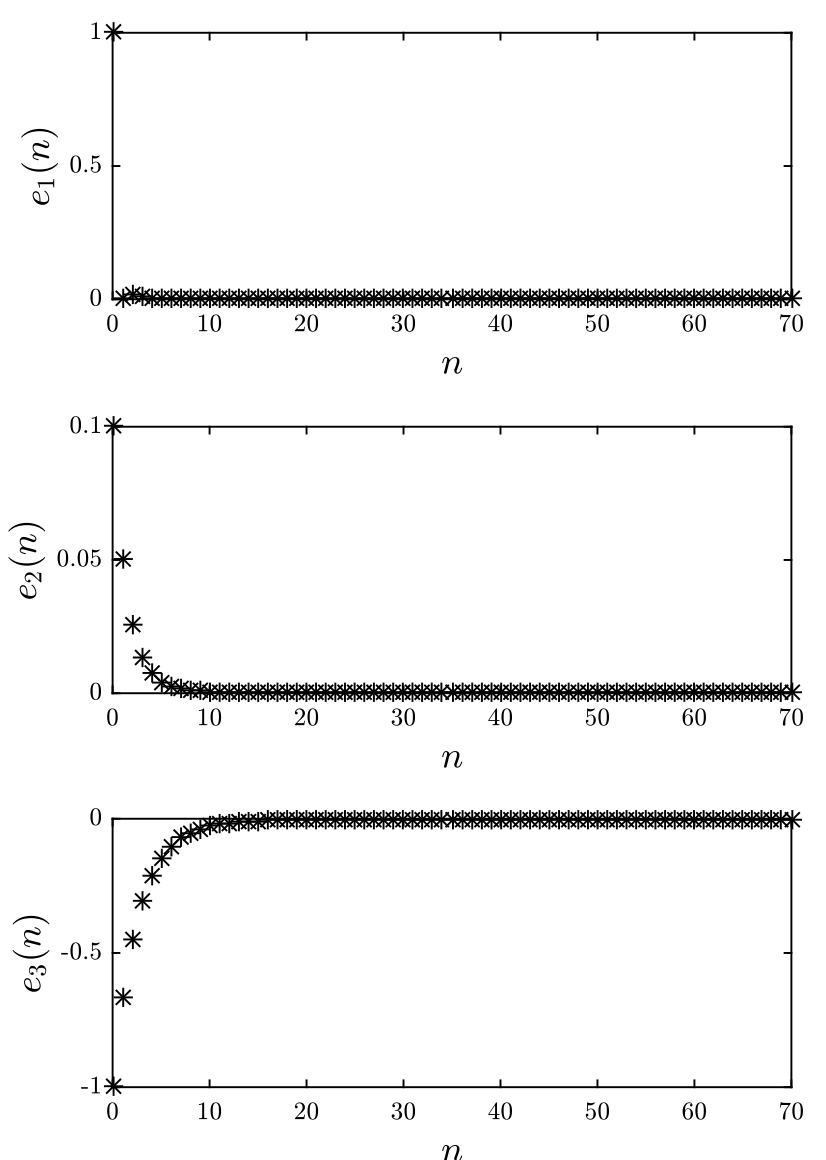

Figure 3 Time evolution of the generalized synchronization errors $e_{1}(t), e_{2}(t)$, and $e_{3}(t)$ with $v=0.984$ and $a=0$

Following the synchronization process described in Sect. 4, the error system is given by

$$
\left(e_{1}(t), e_{2}(t)\right)^{T}=\left(x_{1}(t), x_{2}(t)\right)^{T}-\varphi\left(y_{1}(t), y_{2}(t), y_{3}(t)\right),
$$

where

$$
\varphi\left(y_{1}(t), y_{2}(t), y_{3}(t)\right)=\left(y_{1}(t)-2 y_{2}(t), y_{1}(t)+y_{3}^{2}(t)\right)^{T} .
$$

The function $\varphi$ can be written as

$$
\varphi\left(y_{1}(t), y_{2}(t), y_{3}(t)\right)=H \times\left(y_{1}(t), y_{2}(t), y_{3}(t)\right)^{T}+h\left(y_{1}(t), y_{2}(t), y_{3}(t)\right),
$$

where

$$
H=\left(\begin{array}{ccc}
1 & -2 & 0 \\
0 & 1 & 0
\end{array}\right)
$$

and

$$
h\left(y_{1}(t), y_{2}(t), y_{3}(t)\right)=\left(0, y_{3}^{2}(t)\right)^{T} .
$$



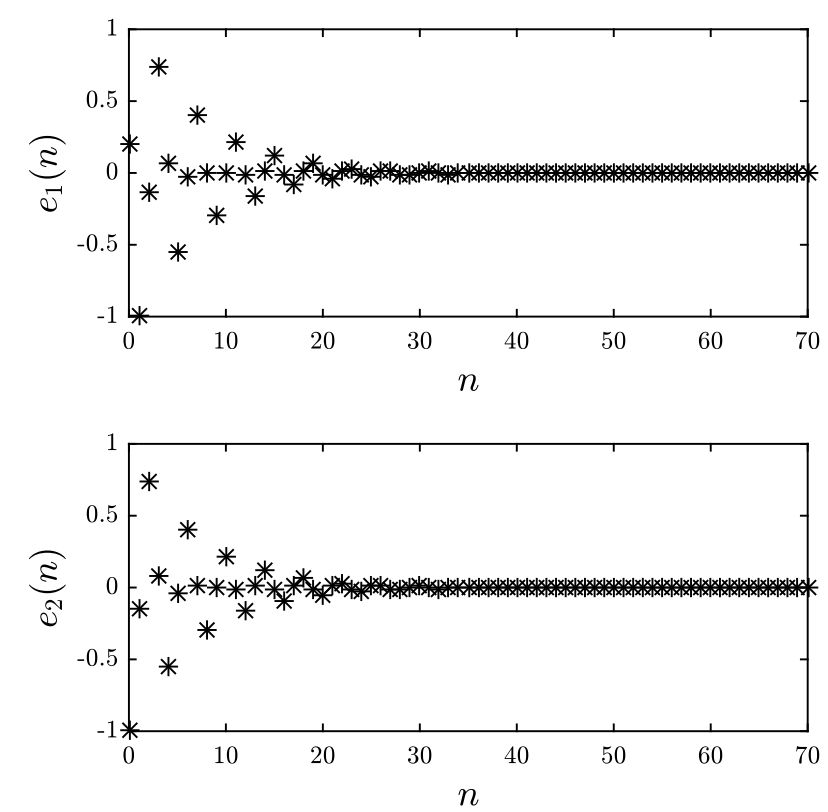

Figure 4 Time evolution of the inverse generalized synchronization errors $e_{1}(t)$ and $e_{2}(t)$ with $v=0.984$ and $a=0$

Using the steps of Sect. 4, we have

$$
\hat{H}=\left(\begin{array}{cc}
1 & -2 \\
0 & 1
\end{array}\right) \quad \text { and } \quad \hat{H}^{-1}=\left(\begin{array}{ll}
1 & 2 \\
0 & 1
\end{array}\right) \text {. }
$$

According to Theorem 4, the control matrix $L$ can be selected as

$$
L=\left(\begin{array}{cc}
0 & 0 \\
b_{1}-1 & -2
\end{array}\right)
$$

which clearly satisfies condition (25). Recall from (26) that the controller requires us to determine $\hat{H}^{-1}$ and $R$, which may be calculated using $L$ as in (42) and $A$ as in (35). Constructing the controllers is, therefore, straightforward and is governed by (22) and (26). As a result, systems (28) and (29) are inverse generalized synchronized in two dimensions. Figure 4 depicts the convergence of the errors, which belong to the fractional system

$$
\left\{\begin{array}{l}
{ }^{C} \Delta_{a}^{v} e_{1}(t)=-e_{1}(t+v-1)+e_{2}(t+v-1), \\
{ }^{C} \Delta_{a}^{v} e_{2}(t)=e_{1}(t+v-1)-e_{2}(t+v-1) .
\end{array}\right.
$$

\subsection{Discussion of results}

It is important to note that the novelty of the results reported in this paper stems from the fact that the GS scheme achieved in this paper is a generalization of a number of different synchronization types including complete synchronization, anti-synchronization, projective synchronization, matrix projective synchronization, and functional matrix projective synchronization. Similarly, a number of different types considered in the literature fall 
under the IGS type including inverse synchronization, anti-synchronization, inverse projective synchronization, inverse matrix projective synchronization, and inverse functional matrix projective synchronization.

The literature related to the synchronization of fractional chaotic maps is still very limited. Perhaps the most relevant studies are [38-41]. In [38], the authors discuss the dynamics of the fractional logistic map and propose a simple synchronization scheme for a pair of identical maps. The work in [39] concerns identical synchronization, which is based on [18], and uses the results reported in [52, 53]. Also, [40] deals with the chaotic synchronization of linearly coupled discrete fractional Hénon maps. The study considers identical drive and response systems and the synchronization scheme achieved is basic. As for [41], the fractional difference operator employed is different from ours, which makes it difficult to compare the results except to say that the Caputo operator used here is more practical for most applications.

Unlike all of the previous studies, our work is more general both in terms of the drive/response pair considered and the types of synchronization achieved. The GS and IGS synchronization schemes proposed here are applicable to a wide range of fractional maps. In addition, the method used to establish asymptotic stability is the linearization scheme proved in [4].

\section{Conclusion and future works}

In this paper, we have proposed control strategies for two distinct synchronization types dedicated to discrete-time fractional-order chaotic systems characterized by different dimensions. The first type is referred to as generalized synchronization and the second as inverse generalized synchronization. The proposed control parameters are nonlinear in nature. In order to ensure that the proposed schemes converge towards zero, we establish the asymptotic stability of the zero solution to the error system by means of the linearization method. In order to assess the validity of the findings, numerical results have been presented for a 2D master and a 3D slave. The discrete-time systems employed here are well known in the literature. Two distinct cases have been considered: a 3D generalized synchronization and a 2D inverse generalized synchronization. Matlab simulation results have confirmed the convergence of the error in sufficient time.

We note that the global asymptotic stability of controllers is usually established by means of the direct Lyapunov method. Unfortunately, since the method has yet to be proven for fractional discrete systems with delay, we were not able to use it in our study. We plan to address this in a future study. In addition, discrete-time fractional chaotic systems have received considerable interest in the field of data, image, and video encryption. It is common to use a large set of random or pseudo-random keys in a secret or public key encryption scenario. The basic idea is that chaotic maps can be used instead of conventional algorithmic key generators. It has been claimed in the literature that the dependence of fractional chaotic maps on changes in the fractional order adds a new degree of freedom, which makes them more suitable for encryption purposes. It is the intention of the authors to put this claim to the test in a future study by comparing the synchronization scheme discussed herein with previous results in a data encryption scenario. 
Funding

The authors declare that no funding has been received for work that resulted in this manuscript.

\section{Competing interests}

The authors declare that they do not have any competing interests in this manuscript.

\section{Authors' contributions}

All authors contributed equally to this manuscript. All authors read and approved the final manuscript.

\section{Author details}

'Department of Mathematics and Computer Sciences, University of Larbi Ben M'hidi, Oum El Bouaghi, Algeria. ${ }^{2}$ Mathematics and Computer Science Department, Tebessa University, Tebessa, Algeria. ${ }^{3}$ Department of Electrical Engineering, College of Engineering, Taibah University, Yanbu, Saudi Arabia. ${ }^{4}$ Dipartimento Ingegneria Innovazione, Universita del Salento, Lecce, Italy. ${ }^{5}$ Institute for Advanced Study, Shenzhen University, Shenzhen, P.R. China. ${ }^{6}$ Modeling Evolutionary Algorithms Simulation and Artificial Intelligent, Faculty of Electrical \& Electronics Engineering, Ton Duc Thang University, Ho Chi Minh City, Vietnam.

\section{Publisher's Note}

Springer Nature remains neutral with regard to jurisdictional claims in published maps and institutional affiliations.

\section{Received: 10 May 2018 Accepted: 15 August 2018 Published online: 31 August 2018}

\section{References}

1. Goodrich, C., Peterson, A.C.: Discrete Fractional Calculus. Springer, Berlin (2015)

2. Baleanu, D., Jajarmi, A., Asad, J., Blaszczyk, T.: The motion of a bead sliding on a wire in fractional sense. Acta Phys. Pol. A 131(6), 1561-1564 (2017)

3. Jajarmi, A., Hajipour, M., Mohammadzadeh, E., Baleanu, D.: A new approach for the nonlinear fractional optimal control problems with external persistent disturbances. J. Franklin Inst. 335(9), 3938-3967 (2018)

4. Cermak, J., Gyori, I., Nechvatal, L.: On explicit stability conditions for a linear fractional difference system. Fract. Calc. Appl. Anal. 18(3), 651-672 (2015)

5. Baleanu, D., Wu, G., Bai, Y., Chen, F.: Stability analysis of Caputo-like discrete fractional systems. Commun. Nonlinear Sci. Numer. Simul. 48, 520-530 (2017)

6. Wu, G.C., Baleanu, D., Luo, W.H.: Lyapunov functions for Riemann-Liouville-like fractional difference equations. Appl. Math. Comput. 314, 228-236 (2017)

7. Wu, G.C., Baleanu, D., Huang, L.L.: Novel Mittag-Leffler stability of linear fractional delay difference equations with impulse. Appl. Math. Lett. 82, 71-78 (2018)

8. Wu, G.C., Baleanu, D.: Stability analysis of impulsive fractional difference equations. Fract. Calc. Appl. Anal. 21, 354-375 (2018)

9. Hénon, M.: A two-dimensional mapping with a strange attractor. Commun. Math. Phys. 50(1), 69-77 (1976)

10. Lozi, R.: Un atracteur étrange du type attracteur de Hénon. J. Phys. (Paris) 39, 9-10 (1978)

11. Itoh, M., Yang, T., Chua, L.O.: Conditions for impulsive synchronization of chaotic and hyperchaotic systems. Int. J. Bifurc. Chaos Appl. Sci. Eng. 11, 551-558 (2001)

12. Hitzl, D.L., Zele, F.: An exploration of the Hénon quadratic map. Phys. D: Nonlinear Phenom. 14(3), 305-326 (1985)

13. Stefanski, K.: Modelling chaos and hyperchaos with 3D maps. Chaos Solitons Fractals 9(1-2), 83-93 (1998)

14. Kapitaniak, T.: Chaos for Engineers: Theory, Applications, and Control. Springer, Berlin (2000)

15. Banerjee, S., Rondoni, L.: Applications of Chaos and Nonlinear Dynamics in Science and Engineering Vol. III. Springer, Berlin (2013)

16. Curry, D.M.: Practical application of chaos theory to systems engineering. Proc. Comput. Sci. 8, 39-44 (2012)

17. Aihara, K.: Chaos and its applications. Proc. IUTAM 5, 199-203 (2012)

18. Pecora, L.M., Carrol, T.L.: Synchronization in chaotic systems. Phys. Rev. A 64, Article ID 821 (1990)

19. Ma, Z., Liu, Z., Zhang, G.: Generalized synchronization of discrete systems. Appl. Math. Mech. 28(5), 609-614 (2007)

20. Ouannas, A., Azar, A.T., Abu-Saris, R.: A new type of hybrid synchronization between arbitrary hyperchaotic maps. Int. J. Mach. Learn. Cybern. 8, 1887-1894 (2017)

21. Ouannas, A., Grassi, G.: A new approach to study co-existence of some synchronization types between chaotic maps with different dimensions. Nonlinear Dyn. 86(2), 1319-1328 (2016)

22. Ouannas, A., Odibat, Z:: Generalized synchronization of different dimensional chaotic dynamical systems in discrete-time. Nonlinear Dyn. 81(1), 765-771 (2015)

23. Ouannas, A.: A new generalized-type of synchronization for discrete chaotic dynamical systems. J. Comput. Nonlinear Dyn. 10(6), Article ID 061019 (2015)

24. Ouannas, A., Grassi, G.: Inverse full state hybrid projective synchronization for chaotic maps with different dimensions. Chin. Phys. B 25(9), Article ID 090503 (2016)

25. Ouannas, A., Odibat, Z., Shawagfeh, N.: Universal chaos synchronization control laws for general quadratic discrete systems. Appl. Math. Model. 45, 636-641 (2017)

26. Ouannas, A., Grassi, G., Karouma, A., Ziar, T., Wang, X., Pham, V.T.: New type of chaos synchronization in discrete-time systems: the F-M synchronization. Open Phys. 16, 174-182 (2018)

27. Grassi, G.: Generalized synchronization between different chaotic maps via dead-beat control. Chin. Phys. B 21(5), Article ID 050505 (2012)

28. Edelman, M., Tarasov, V.E.: Fractional standard map. Phys. Lett. A 374, 279-285 (2009)

29. Mumkhamar, J.: Chaos in a fractional order logistic map. Fract. Calc. Appl. Anal. 26, 511-519 (2013)

30. Wu, G.C., Baleanu, D.: Discrete fractional logistic map and its chaos. Nonlinear Dyn. 75, 283-287 (2014) 
31. Wu, G.C., Baleanu, D., Zeng, S.D.: Discrete chaos in fractional sine and standard maps. Phys. Lett. A 378, 484-487 (2014)

32. Liu, Y.: Discrete chaos in fractional Hénon maps. Int. J. Nonlinear Sci. 18(3), 170-175 (2014)

33. $\mathrm{Hu}, \mathrm{T}$ : Discrete chaos in fractional Hénon map. Appl. Math. 5, 2243-2248 (2014)

34. Xiao, H., Ma, Y., Li, C.P.: Chaotic vibration in fractional maps. J. Vib. Control 20, 964-972 (2014)

35. Liu, C.X., Huang, L.L., Wu, K.T.: Chaos in discrete fractional cubic logistic map and bifurcation analysis. J. Comput. Complex. Appl. 1(2), 105-111 (2015)

36. Huang, L.L., Baleanu, D., Wu, G.C., Zeng, S.D.: A new application of the fractional logistic map. Rom. J. Phys. 61(7-8), 1172-1179 (2016)

37. Kassim, S., Hamiche, H., Djennoune, S., Bettayeb, M.: A novel secure image transmission scheme based on synchronization of fractional-order discrete-time hyperchaotic systems. Nonlinear Dyn. 88, 2473-2489 (2017)

38. Wu, G., Baleanu, D.: Chaos synchronization of the discrete fractional logistic map. Signal Process. 102, 96-99 (2014)

39. Wu, G., Baleanu, D., Xie, H., Chen, F.: Chaos synchronization of fractional chaotic maps based on the stability condition. Physica A 460, 374-383 (2016)

40. Liu, Y: Chaotic synchronization between linearly coupled discrete fractional Hénon maps. Indian J. Phys. 90, 313-317 (2016)

41. Megherbi, O., Hamiche, H., Djennoune, S., Bettayeb, M.: A new contribution for the impulsive synchronization of fractional-order discrete-time chaotic systems. Nonlinear Dyn. 90, 1519-1533 (2017)

42. Xin, B., Liu, L., Hou, G., Ma, Y.: Chaos synchronization of nonlinear fractional discrete dynamical systems via linear control. Entropy 19, Article ID 351 (2017)

43. Jajarmi, A., Hajipour, M., Baleanu, D.: New aspects of the adaptive synchronization and hyperchaos suppression of a financial model. Chaos Solitons Fractals 99, 285-296 (2017)

44. Zhang, G., Liu, Z., Ma, Z.: Generalized synchronization of different dimensional chaotic dynamical systems. Chaos Solitons Fractals 32, 773-779 (2007)

45. Terry, J.R., VanWiggeren, G.D.: Chaotic communication using generalized synchronization. Chaos Solitons Fractals 12(1), 145-152 (2001)

46. Shukla, M.K., Sharma, B.B.: Investigation of chaos in fractional order generalized hyperchaotic Hénon map. Int. J. Electron. Commer. 78, 265-273 (2017)

47. Atici, F.M., Eloe, P.W.: Discrete fractional calculus with the nabla operator. Electron. J. Qual. Theory Differ. Equ., Spec. Ed. I 2009, Article ID 3 (2009)

48. Abdeljawad, T.: On Riemann and Caputo fractional differences. Comput. Math. Appl. 62, 1602-1611 (2011)

49. Hajipour, M., Jajarmi, A., Baleanu, D.: An efficient nonstandard finite difference scheme for a class of fractional chaotic systems. J. Comput. Nonlinear Dyn. 13(2), Article ID 021013 (2017)

50. Anastassiou, G.A.: Principles of delta fractional calculus on time scales and inequalities. Math. Comput. Model. 52, 556-566 (2010)

51. Ouannas, A.: On inverse generalized synchronization of continuous chaotic dynamical systems. Int. J. Appl. Comput. Math. 2(1), 1-11 (2016)

52. Abu-Saris, R., Al-Mdallal, Q.: On the asymptotic stability of linear system of fractional order difference equations. Fract. Calc. Appl. Anal. 16, 613-629 (2013)

53. Mozyrska, D., Wyrwas, M.: The Z-transform method and delta type fractional difference operators. Discrete Dyn. Nat. Soc. 2013, Article ID 852734 (2013)

\section{Submit your manuscript to a SpringerOpen ${ }^{\circ}$ journal and benefit from:}

- Convenient online submission

- Rigorous peer review

- Open access: articles freely available online

- High visibility within the field

- Retaining the copyright to your article

Submit your next manuscript at $\boldsymbol{~ s p r i n g e r o p e n . c o m ~}$ 